\title{
AVALIAÇÃO NUTRICIONAL DE PACIENTES COM CIRROSE PELO VÍRUS DA HEPATITE C: a aplicação da calorimetria indireta
}

\author{
Catarina Bertaso Andreatta GOTTSCHALL, Mário Reis ÁLVARES-da-SILVA, \\ Ana Cristina Riehs CAMARGO, Renata Medeiros BURTETT e Themis Reverbel da SILVEIRA
}

RESUMO - Racional - A desnutrição é freqüente em cirróticos, mas sua avaliação é difícil. A predição do gasto energético basal pela equação de Harris-Benedict não está validada neste grupo. A alternativa seria mensurá-la através da calorimetria indireta. Objetivos - Realizar avaliação nutricional em cirróticos, aferir o gasto energético basal pela calorimetria indireta e compará-lo ao estimado pela equação de Harris-Benedict. Material e Métodos - Estudaram-se 34 adultos com cirrose pelo vírus da hepatite C, em acompanhamento ambulatorial, classificados de acordo com Child-Pugh e escore "model of end-stage liver disease". O gasto energético basal foi estimado pela equação de Harris-Benedict e medido pela calorimetria indireta. Avaliação nutricional foi realizada por antropometria, avaliação nutricional subjetiva global, dinamometria e inquérito recordatório. Resultados - Em relação à classificação de Child-Pugh, 15 (44,2\%) eram A, 12 (35,3\%) B e 7 (20,6\%) C e 33 $(97,1 \%)$ apresentaram valores inferiores a 20 no escore "model of end-stage liver disease". O gasto energético basal estimado foi maior do que o medido (Harris-Benedict 1404,5 $\pm 150,3 \mathrm{kcal}$; calorimetria indireta 1059,9 \pm 309,6 kcal). A prevalência de desnutrição variou entre os métodos (índice de massa corpórea, circunferência muscular do braço, avaliação nutricional subjetiva global, prega cutânea do tríceps e dinamometria: $0 \% ; 5,9 \% ; 17,6 \% ; 35,3 \%$ e $79,4 \%$, respectivamente). A ingestão calórica e protéica foi de $80 \%$ e $85 \%$ do recomendado, com inadequação na ingestão de cálcio, magnésio, ferro e zinco. Conclusões - A desnutrição foi freqüente. A dinamometria parece ser o melhor método para o diagnóstico. A ingestão foi inadequada. Considerando que o gasto energético basal estimado foi superior ao medido e a necessidade de maior aporte calórico, a utilização da equação de predição talvez possa substituir a calorimetria indireta.

DESCRITORES - Cirrose hepática. Hepacivírus. Desnutrição. Avaliação nutricional. Metabolismo energético. Calorimetria indireta. Força da mão.

\section{INTRODUÇÃO}

A cirrose é uma doença hepática crônica, caracterizada por processo difuso que envolve fibrose, alteração na arquitetura lobular e regeneração nodular ${ }^{(36)}$. A principal causa de cirrose no mundo ocidental é a infecção crônica pelo vírus da hepatite $\mathrm{C}$ (VHC), associada ou não ao consumo excessivo de álcool ${ }^{(38)}$.

A desnutrição é freqüentemente encontrada nos pacientes com hepatopatia crônica de origem alcoólica ou não-alcoólica ${ }^{(4,}$ $21,22,23,24,25,27)$, podendo advir de baixa ingestão alimentar, alteração na síntese, metabolismo e armazenamento de nutrientes, má-digestão e absorção de nutrientes ou ainda, ser secundária à hipermetabolismo ${ }^{(18,35)}$.

A avaliação do estado nutricional deve ser realizada sistematicamente em todo paciente com doença hepática ${ }^{(2)}$. Os métodos disponíveis são anamnese alimentar, antropometria, métodos bioquímicos e imunológicos e testes compostos ${ }^{14,15}$, 16). Com freqüência, são de difícil valorização nesta população por peculiaridades da doença hepática. A avaliação funcional da nutrição através da dinamometria é simples, barata e eficaz, sendo um método que poderia minimizar as dificuldades na avaliação nutricional em hepatopatas.
O gasto energético basal (GEB) é a quantidade de oxigênio consumida durante o repouso, em jejum. A predição do GEB através de equação padrão de Harris-Benedict (HB), pode trazer resultados seguros em determinadas populações ${ }^{(12)}$, mas não está validada para pacientes com cirrose, já que existe grande variação da mensuração do GEB em indivíduos cirróticos, quando comparados a indivíduos normais ${ }^{(19)}$. Uma alternativa é sua mensuração através da calorimetria indireta $(\mathrm{CI})^{(17)}$, método que permite determinar com maior precisão o estado metabólico ${ }^{(41)}$. Não há, de acordo com o conhecimento dos autores deste trabalho, estudo em nosso meio que tenha aferido o GEB em indivíduos cirróticos pelo VHC, utilizando a calorimetria indireta, tampouco comparando-a à avaliação nutricional através da dinamometria.

\section{MATERIAL E MÉTODOS}

Realizou-se estudo transversal em pacientes com mais de 18 anos, com cirrose secundária ao VHC, em acompanhamento ambulatorial no Serviço de Gastroenterologia do Hospital de Clínicas de Porto Alegre (HCPA), RS. O diagnóstico de cirrose foi baseado em testes clínicos, laboratoriais, radiológicos e, em

Programa de Pós Graduação: Ciências em Gastroenterologia, Faculdade de Medicina, Universidade Federal do Rio Grande do Sul, Porto Alegre, RS.

Endereço para correspondência: Nutr. Catarina Bertaso Andreatta Gottschall - Rua Desembargador Augusto Loureiro Lima, 129 - apt. 402 - Bairro Petrópolis - $90470-120$ - Porto

Alegre, RS. E-mail: cgottschall@hcpa.ufrgs.br 
alguns casos, histológicos. Excluíram-se pacientes com hepatocarcinoma e aqueles com histórico de consumo excessivo de álcool, bem como os indivíduos sem condições de usar o dinamômetro e aqueles com retardo mental ou alterações neuromusculares nos membros superiores.

A gravidade da doença hepática foi avaliada pela classificação de ChildPugh $^{(32)}$ e pelo escore MELD ("model of end-stage liver disease") ${ }^{(7,36)}$.

A coleta de dados foi realizada de janeiro a abril de 2002, sendo estudadas a mensuração do GEB através da calorimetria indireta (CI) a estimação do GEB pela equação de HB e avaliação nutricional. Os indivíduos foram recrutados na primeira consulta do ambulatório da nutricionista responsável pelo projeto e compareceram na data e local agendados entre 7 e 9 horas da manhã, em jejum de 12 horas, para realizar os procedimentos.

Todos os indivíduos concordaram em participar do estudo e assinaram o termo de consentimento informado, aprovado pelo Comitê de Ética em Pesquisa do HCPA.

\section{Mensuração da taxa metabólica basal (TMB)}

A medição da TMB foi realizada pela manhã, em sala com temperatura ambiente controlada, baixa luminosidade e sem ruídos, com o indivíduo em decúbito dorsal, após repouso de 20 minutos. Era fixada em seu rosto uma máscara conectada ao calorímetro portátil (Teen 100 - Aerosport Inc., Ann Arbor, Michigan, EUA). O equipamento foi calibrado antes e após cada medida. O consumo de oxigênio $\left(\mathrm{VO}_{2}\right)$ e a produção de gás carbônico $\left(\mathrm{VCO}_{2}\right)$ eram medidos durante 25 minutos com o indivíduo permanecendo deitado e sem se movimentar. A medida da TMB/minuto em quilocaloria ( $\mathrm{kcal} / \mathrm{min})$ era obtida através da equação:

$$
\mathrm{kcal} / \mathrm{min}=\left\{\left[3,9\left(\mathrm{VO}_{2}\right)\right]+\left[1,1\left(\mathrm{VCO}_{2}\right)\right]\right\}
$$

descrita por WEIR ${ }^{(43)}$, com os últimos 20 minutos, observando tempo de equilíbrio inicial de 5 minutos, cuja média foi multiplicada por 1440 para se obter a TMB de 24 horas.

\section{Equação de predição}

A equação de predição de HB foi utilizada para estimar o gasto energético basal de indivíduos enfermos. Esta equação desenvolvida, uma para cada sexo, inclui como variáveis independentes a massa corporal, a estatura e a idade ${ }^{(12)}$.

\section{Avaliação do Estado Nutricional}

A avaliação nutricional incluiu avaliação nutricional subjetiva global (ANSG) $)^{(8,10)}$, aferição da força do aperto de mão nãodominante por dinamometria $(\mathrm{FAM})^{(2,42)}$, cujos resultados foram comparados a valores de referência na população deste estudo ${ }^{3}$, ${ }^{5)}$, inquérito alimentar de $3 \operatorname{dias}^{(34)}$ e antropometria (peso e altura, índice de massa corporal -IMC, prega cutânea do tríceps - $\mathrm{PCT}$ e circunferência muscular do braço $-\mathrm{CMB})^{(13)}$.

\section{Cálculo da amostra}

O cálculo da amostra foi realizado através do programa estatístico "Computer Programs for Epidemiologists" (PEPI), versão 3.0 , com poder de $80 \%$, intervalo de confiança de $95 \%$ e nível de significância de 0,05 .

O tamanho da amostra foi determinado em 33 indivíduos, considerando uma diferença de 250 calorias no GEB medido e estimado, e desvio padrão de 320 e 360 calorias.

\section{Análise dos dados}

A apresentação da amostra foi descritiva, na forma de tabelas, como média, desvio padrão e freqüência. As comparações entre GEB medido pela calorimetria e o GEB estimado pela equação de predição, seguiram o método proposto por BLAND e ALTMAN ${ }^{(6)}$ com estimativa da diferença média e seu limite de concordância de 95\% (LC 95\%). O nível de significância adotado foi de $5 \%(\alpha=0,05)$. A análise dos dados foi realizada pelo programa estatístico "Statistical Package for the Social Sciences", versão 10.0 (SPSS Inc, Chicago, IL, EUA). A comparação das médias entre o estado nutricional avaliado pela FAM e ANSG e o GEB foi realizado pelo teste $t$ de Student para amostras independentes.

\section{RESULTADOS}

A amostra foi composta por 34 indivíduos com cirrose pelo vírus C, sendo $18(52,9 \%)$ homens e $16(47,1 \%)$ mulheres, com média de idade de 53,7 anos (41 a 70 anos).

Quanto ao grau de doença hepática, 15 pacientes $(44,2 \%)$ foram classificados como Child-Pugh A, 12 (35,3\%) como B e 7 (20,6\%) como C. Apenas quatro (11,8\%) indivíduos apresentaram ascite. Em relação ao escore MELD, 33 (97,1\%) apresentaram valores inferiores a 20. As características clínicas da população estudada estão descritas na Tabela 1 .

TABELA 1 - Características dos 34 pacientes analisados

\begin{tabular}{lc}
\hline Características & Valores \\
\hline Gênero, $\mathrm{n}(\%)$ & $18(52,9)$ \\
Masculino & $16(47,1)$ \\
Feminino & $53,67 \pm 7,68$ \\
Idade, anos & \\
Classificação de Child-Pugh, n (\%) & $15(44,1)$ \\
A & $12(35,3)$ \\
B & $7(20,6)$ \\
C & \\
MELD, n (\%) & $12(35,3)$ \\
$<10$ & $21(61,8)$ \\
$10-19$ & $1(2,9)$ \\
$20-29$ &
\end{tabular}

Vinte e dois indivíduos $(64,7 \%)$ responderam e entregaram o inquérito recordatório e em 19 (55,9\%) foi possível utilizar os dados da calorimetria indireta. Nos 15 pacientes restantes, os níveis de $\mathrm{VO}_{2}$ e $\mathrm{VCO}_{2}$ encontravamse abaixo do recomendado, o que possibilitaria a alteração da qualidade dos dados da calorimetria, motivo pelo qual foram excluídos.

A média da ingestão calórica em 22 indivíduos foi de $1613 \pm$ $374,7 \mathrm{kcal} /$ dia, com consumo médio de $60,9 \pm 20,6 \mathrm{~g}$ proteínas/dia. $\mathrm{Na}$ Tabela 2 estão discriminados os valores médios de ingestão diária de macro e micronutrientes, bem como o percentual do ideal para cada indivíduo de acordo com sexo, idade e gravidade da doença $\mathrm{a}^{(12,25,30)}$.

TABELA 2 - Ingestão diária de macro e micronutrientes: média e relação com o percentual ideal $(n=22)$

\begin{tabular}{lcc}
\hline Variável & Média $\pm \mathrm{DP}$ & \% do ideal \\
\hline Quilocaloria, kcal & $1613 \pm 374,7$ & 80,6 \\
Proteína, g & $61 \pm 20,6$ & 85,9 \\
Lipídios, g & $52,7 \pm 15,6$ & Adequado \\
Carboidratos, $\mathrm{g}$ & $235,4 \pm 67,3$ & Adequado \\
Cálcio, $\mathrm{mg}$ & $647,4 \pm 281,4$ & $<75$ \\
Fósforos, mg & $902,5 \pm 378,8$ & Adequado \\
Magnésio, $\mathrm{mg}$ & $178,5 \pm 85,5$ & $<60$ \\
Ferro, $\mathrm{mg}$ & $10,1 \pm 4,6$ & $<85$ \\
Sódio, $\mathrm{mg}$ & $1441,2 \pm 492,2$ & $>200$ \\
Potássio, $\mathrm{mg}$ & $2296,1 \pm 1031,4$ & Adequado \\
Zinco, $\mathrm{mg}$ & $4,5 \pm 2,2$ & $<35$ \\
\hline Onde: \% ideal considerando: $30 \mathrm{kcal} / \mathrm{kg}, 1 \mathrm{~g}$ proteína $/ \mathrm{kg}, 25 \%$ a $40 \%$ lipídios, $70 \%$ a $65 \%$ carboidratos.
\end{tabular}

Onde: \% ideal considerando: $30 \mathrm{kcal} / \mathrm{kg}, 1 \mathrm{~g}$ proteína $/ \mathrm{kg}, 25 \%$ a $40 \%$ lipídios, $70 \%$ a $65 \%$ carboidratos Micronutrientes segundo RDA. 
Os parâmetros nutricionais estão descritos na Tabela 3. A média de peso dos indivíduos foi de $70,5 \mathrm{~kg}$ e a estatura média de $163 \mathrm{~cm}$, o que correspondeu ao índice de massa corporal médio de $26,6 \mathrm{~kg} / \mathrm{m}^{2}$, onde 13 $(38,2 \%)$ encontravam-se eutróficos e $21(61,8 \%)$ com sobrepeso. Quando avaliados pela ANSG, $22(64,7 \%)$ pacientes estavam bem nutridos e 12 $(35,3 \%)$ com desnutrição leve. Na avaliação da PCT, seis $(17,6 \%)$ pacientes tinham algum grau de desnutrição e na CMB dois (5,9\%) encontravam-se desnutridos. A mensuração da FAM pela dinamometria demostrou que 27 pacientes $(79,4 \%)$ encontravam-se em risco nutricional e apenas 7 pacientes bem nutridos. Em relação à desnutrição e gravidade da doença hepática, a FAM detectou desnutrição em 10 pacientes Child A $(66,7 \%)$ e em 17 Child B e C (84,5\%), enquanto que a ANSG detectou desnutrição em 3 pacientes Child A (20\%) e em 9 pacientes Child B e C (47,4\%).

TABELA 3 - Características nutricionais da amostra estudada

\begin{tabular}{lcc}
\hline Variável & Média \pm DP & Desnutrição \\
\hline Estatura $(\mathrm{cm})$ & $163 \pm 85$ & - \\
Peso $(\mathrm{kg})$ & $70,5 \pm 12,56$ & - \\
$\mathrm{IMC}(\mathrm{kg} / \mathrm{m} 2)$ & $26,63 \pm 4,47$ & $0(0,0)$ \\
CMB & $25,43 \pm 3,93$ & $2(5,9)$ \\
PCT $(\mathrm{cm})$ & $17,68 \pm 6,55$ & $6(17,6)$ \\
ANSG & - & $12(35,3)$ \\
FAM $(\mathrm{kg} / \mathrm{f})$ & $23,45 \pm 10,84$ & $27(79,4)$ \\
\hline
\end{tabular}

Os dados são apresentados como média \pm desvio-padrão e freqüência (\%)

IMC: índice de massa corporal

CMB: circunferência muscular do braço

PCT: prega cutânea triciptal

FAM: foçaçaçäo nutricional subjetiva globa

O valor médio $( \pm \mathrm{DP})$ da TMB medida foi de $1059,9 \pm 309,6 \mathrm{kcal}$ em 19 pacientes, enquanto que a média do valor estimado pela equação foi de $1404,5 \pm 150,3 \mathrm{kcal}$.

Os LC de 95\% entre a TMB medida e estimada estão demonstrados no gráfico de BLAND e ALTMAN (Figura 1). A variação do LC 95\% foi de 123 a $812 \mathrm{kcal}$, com diferença média de $344 \mathrm{kcal}$ a mais na TMB estimada por HB.

Na Tabela 4, são comparados os achados da TMB estimada e medida em relação à avaliação nutricional por FAM e ANSG.

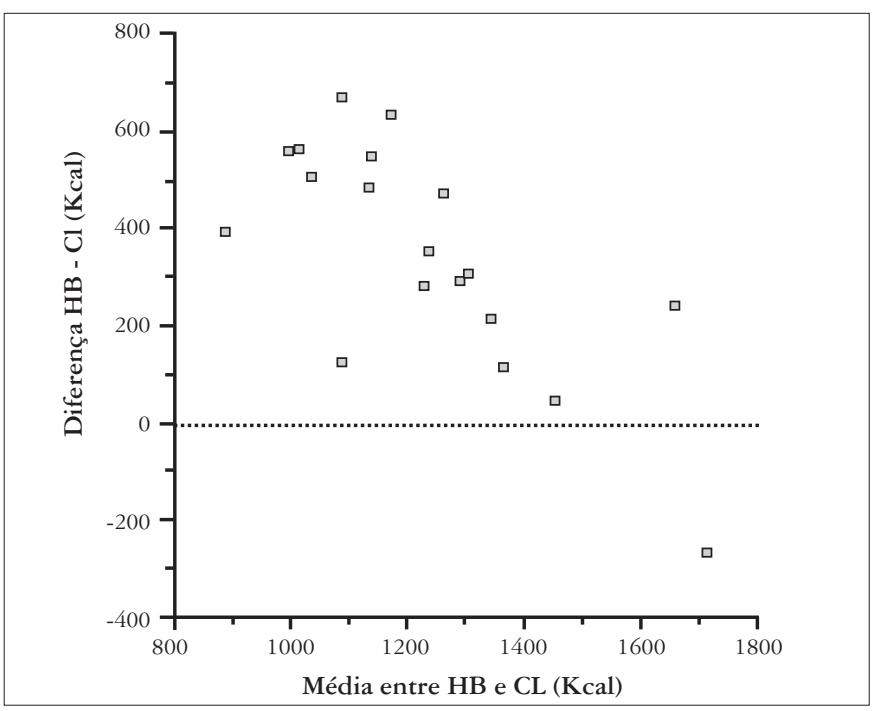

FIGURA 1 - Gráfico de Bland-Altman representando a diferença entre o gasto energético basal estimado pela equação $\mathrm{HB}$ e medido pela calorimetria indireta $(\mathrm{HB}-\mathrm{CI})$ versus a média $((\mathrm{HB}+\mathrm{CI} / 2), \mathrm{n}=19$
TABELA 4 - Comparação entre estado nutricional e gasto energético basal

\begin{tabular}{|c|c|c|c|c|}
\hline \multirow[b]{2}{*}{ Avaliação nutricional } & \multicolumn{2}{|c|}{ GEB HB (kcal) } & \multicolumn{2}{|c|}{ GEB CI (kcal) } \\
\hline & $\mathrm{n}$ & valor & $\mathrm{n}$ & valor \\
\hline \multicolumn{5}{|l|}{ FAM } \\
\hline Desnutrido & 27 & $1385,0 \pm 142,1$ & 14 & $1015,2 \pm 261,0$ \\
\hline Bem nutrido & 7 & $1416,9 \pm 155,5$ & 5 & $1185,2 \pm 428,1$ \\
\hline Diferença & & $-31,8$ & & -170 \\
\hline IC95\% & & $-156,9$ a 93,2 & & $-509,2$ a 169,2 \\
\hline $\mathrm{P}$ & & 0,608 & & 0,792 \\
\hline
\end{tabular}

ANSG

$\begin{array}{lcccc}\text { Desnutrido } & 12 & 1423,7 \pm 118,3 & 6 & 1088,6 \pm 280,5 \\ \text { Bem nutrido } & 22 & 1374,1 \pm 154,8 & 13 & 1046,7 \pm 332,3 \\ \text { Diferença } & & 49,6 & & 42,0 \\ \text { IC } 95 \% & -55,1 \text { a } 154,3 & & -289,1 \text { a } 373,0 \\ \text { P } & 0,342 & & 0,792\end{array}$

GEB HB: gasto energético basal estimado pela equação de Harris-Benedict

GEB Cl: gasto energético basal medido pela calorimetria indireta

FAM: força do aperto da mão-não-dominante

ANSG: avaliação nutricional subjetiva globa

\section{DISCUSSÃO}

A desnutrição é freqüentemente encontrada em pacientes com doença hepática crônica e pode influenciar na sobrevida a curto e longo prazo ${ }^{(2)}$. A maior parte dos estudos avalia pacientes cirróticos independente da etiologia, estudando etiologia viral (vírus B, vírus C), colestática, alcoólica e outras ${ }^{(1,2,11)}$, o que é inadequado. Existem poucos estudos onde a prevalência de desnutrição é descrita em cirróticos de origem não-alcoólica ${ }^{(30,40)}$ e pouco é sabido da avaliação nutricional e do gasto energético basal em pacientes com cirrose secundária à infecção crônica pelo VHC, o que foi avaliado no presente estudo. Os indivíduos estudados eram pacientes cirróticos ambulatoriais, em sua maioria Child A e B e sem risco de vida apreciável em 3 meses, conforme atestado pelo escore MELD.

A avaliação nutricional é difícil em pacientes cirróticos, os estudos sugerem não haver um padrão-ouro para diagnosticar sua desnutrição.

Nenhum paciente foi considerado desnutrido pelo IMC. Ao contrário, a prevalência de sobrepeso foi alta $(62 \%)$, talvez influenciada pela retenção hídrica. Nos pacientes classificados com sobrepeso pelo IMC, encontrou-se diagnóstico de desnutrição pela ANSG em $38 \%$ e pela FAM $85,7 \%$, portanto, o IMC não parece adequado para avaliar este grupo, o que está de acordo com estudos recentes ${ }^{(33,44,45)}$.

Na ANSG, encontrou-se prevalência de desnutrição de 35,3\%. Alguns componentes da ANSG são qualitativos, como nível de massa muscular e perda de gordura subcutânea, o que favorece esta avaliação, mas em contrapartida, a estimativa de perda de peso que faz parte da avaliação é difícil de ser determinada na presença de ascite e retenção hídrica ${ }^{(1)}$. Vários estudos utilizam a ANSG na avaliação nutricional em cirróticos, apresentando a desnutrição por ela avaliada e a evolução clínica de pacientes ${ }^{(31)}$.

Nos pacientes estudados, a prevalência de desnutrição pelos métodos de PCT e CMB foi baixa - 5,9 e 17,6\%, respectivamente, indo ao encontro com outros estudos ${ }^{(1,2)}$, mas contra outros ${ }^{(33,36)}$.

A utilidade das proteínas séricas é limitada pela diminuição da síntese hepática, o que dificulta sua aplicação na avaliação nutricional. Ao contrário de outros estudos em que foram utilizadas proteínas séricas ${ }^{(33,38,44)}$, como albumina e pré-albumina, este parâmetro não foi avaliado neste trabalho. Boa alternativa para avaliar a desnutrição protéica é a FAM. No presente estudo ela detectou $79,4 \%$ de desnutrição, o que vai de encontro com outros estudos em cirróticos ${ }^{(3,4)}$. Ademais, estudo realizado no nosso meio em 50 pacientes com cirrose, igualmente demonstrou superioridade da FAM no diagnóstico de desnutrição, quando comparado a outros métodos ${ }^{(4)}$. O 
uso da FAM aumentou consideravelmente o diagnóstico de desnutrição quando comparado à ANSG (79,4 e 35,3\%, respectivamente), refletindo provavelmente, a capacidade de diagnosticar a desnutrição em indivíduos sem quaisquer evidências clínicas.

Em cirróticos, uma simples noite de jejum pode ter efeito negativo na homeostase e metabolismo energético e induzir a quebra das reservas endógenas de gordura e proteínas ${ }^{(26)}$. Nos pacientes estudados, a ingestão dietética foi inapropriada tanto em macro, quanto em alguns micronutrientes (Tabela 2). A ingestão calórica dos indivíduos ficou em $80 \%$ do recomendado, o que certamente contribui para um estado nutricional deletério. Achados semelhantes foram descritos por DAVIDSON et al. ${ }^{(9)}$, estudando 52 pacientes cirróticos e comparados a controles. Outro estudo recente em nosso meio ${ }^{(46)}$ avaliou a ingestão de 32 pacientes cirróticos internados, com resultados semelhantes. Em relação aos micronutrientes, encontrou-se inadequação em relação à ingestão de cálcio, de magnésio, de ferro e, especialmente, de zinco.

A baixa ingestão alimentar é freqüentemente observada em pacientes cirróticos, independente do estágio da doença. A impressão que se tem é que hábitos culturais e a falta de orientação especializada induzem a restrições desnecessárias de proteínas e de gorduras. A restrição de sal e a conseqüente redução da palatabilidade dos alimentos é mais um fator que interfere na ingestão. Outro fator que contribui para a inapetência e anorexia destes pacientes é a baixa ingestão de zinco, que tem sido associada a menor acuidade do paladar ${ }^{(20)}$. Na presente série observou-se que todos os pacientes tinham ingestão média de zinco abaixo de 35\% da RDA.

O GEB foi medido pela calorimetria indireta nos 34 indivíduos estudados. Foi possível utilizar seu resultado em 19 pacientes. Nos 15 restantes, o equipamento de medição não conseguiu captar adequadamente as trocas gasosas, reduzindo seus valores e alterando a qualidade dos dados.
No presente estudo o GEB medido pela calorimetria indireta, foi mais baixo que o estimado pela equação de $\mathrm{HB}$, o que está de acordo com alguns estudos prévios ${ }^{(28,37)}$. Opiniões diferentes foram descritas por outros autores que encontraram aumento do GEB medido em relação ao estimado $^{(19,40)}$. Essas observações controversas podem ser explicadas pela heterogeneidade da população de pacientes, por problemas metodológicos associados à medição do GEB e da avaliação nutricional(40).

Alguns estudos descrevem a presença de hipermetabolismo em cirróticos desnutridos. No estudo em questão não houve diferença significativa entre o GEB medido e estimado em pacientes cirróticos bem nutridos e desnutridos.

$\mathrm{Na}$ análise pelo método de BLAND e ALTMAN ${ }^{(6)}$ os dados obtidos entre o GEB estimado e medido não concordam entre si (Figura 1). Estes resultados necessitam de confirmação com amostras maiores.

\section{CONCLUSÕES}

A desnutrição foi freqüente nos pacientes cirróticos estudados e a FAM parece ser o método mais sensível para o seu diagnóstico. A ingestão calórico-protéica foi inadequada nesses pacientes. Considerando que o GEB estimado foi superior ao medido e a necessidade de oferecer um maior aporte calórico a estes pacientes, a utilização da equação de predição talvez possa substituir o uso da calorimetria indireta nestes pacientes.

\section{AGRADECIMENTO}

Ao Fundo de Incentivo a Pesquisa e Eventos (FIPE) do Hospital de Clínicas de Porto Alegre, pelo apoio financeiro.

Gottschall CBA, Álvares-da-Silva MR, Camargo ACR, Burtett RM, Silveira TR. Nutritional assessment in patients with cirrhosis: the use of indirect calorimetry. Arq Gastroenterol 2004;41(4):220-4.

ABSTRACT - Background - Malnutrition is frequent in cirrhotic patients, and its assessment is difficult. Functional assessment through a dynamometer is a simple method and could minimize these drawbacks. Harris-Benedict prediction formulae estimates the resting energy expenditure but has not been validated for this population. One alternative is the use of indirect calorimetry. Aim - To assess nutritional status in cirrhotic patients and estimates the resting energy expenditure through indirect calorimetry and compares it to Harris-Benedict. Patients and Methods - Thirty four adult hepatitis C cirrhotic outpatients were studied, classified by Child-Pugh and model of end-stage liver disease score. The resting energy expenditure was predicted through Harris-Benedict and measured by indirect calorimetry. Nutritional assessment was done through anthropometry, subjective global assessment, hand-grip strength and a 3-day recall. Results - Fifteen (44.2\%) were Child-Pug A, 12 (35.3\%) B and 7 (20.6\%) C, and $33(97.1 \%)$ had model of end-stage liver disease scores less than 20. The resting energy expenditure predicted was higher than the measured (Harris-Benedict $1404.5 \pm 150.3 \mathrm{kcal}$; indirect calorimetry $1059.9 \pm 309.6 \mathrm{kcal}$ ). The prevalence of malnutrition varied between methods (body mass index, muscle arm circumference, subjective global assessment, triceps skinfold thickness and hand-grip strength: $0 ; 5.9 ; 17.6 ; 35.3$ and $79.4 \%$, accordingly). Calories and proteins intake were $80 \%$ and $85 \%$ of recommended amounts and there was inadequate intake of calcium, magnesium, iron and zinc. Conclusions - Malnutrition was frequent and hand-grip strength seemed to be the most sensitive method for its diagnosis. Calories and protein intakes were inadequate. Considering that the predicted resting energy expenditure was higher than the measured one and the need to offer higher caloric intake, the use of the predicting equation may replace indirect calorimetry.

HEADINGS - Liver cirrhosis. Hepacivirus. Malnutrition. Nutrition assessment. Energy metabolism. Calorimetry, indirect. Hand strength. 


\section{REFERÊNCIAS BIBLIOGRÁFICAS}

1. Abbott WJ, Thomson A, Steadman C, Gatton ML, Bothwell C, Kerlin P, Wall DR, Lynch SV. Child-Pugh class, nutritional indicators and early liver transplant outcomes. Hepatogastroenterology 2001;48:823-7.

2. Alberino F, Gatta A, Amodio P, Merkel C, Di Pascoli L, Boffo G, Caregaro L. Nutrition and survival in patients with liver cirrhosis. Nutrition 2001;17:445-50.

3. Álvares-da-Silva MR. Comparação entre a avaliação nutricional subjetiva global, índice nutricional prognóstico e força do aperto da mão não-dominante na avaliação nutricional do paciente cirrótico ambulatorial: a contribuição da dinamometria [dissertação]. Porto Alegre: Universidade Federal do Rio Grande do Sul; 1995.

4. Álvares-da-Silva MR, Gottschall CA, Pruineli RD, Pinto RD, Waechter FL, Cardoso F, Sampaio JA, Smith MM, Francisconi CFM, Pereira-Lima LM. Nutritional evaluation in liver transplantation [abstract]. Hepatology 1998;28(4):746(A).

5. Álvares-da-Silva MR, Silveira TR. O estudo da força do aperto de mão nãodominante em indivíduos sadios. Determinação dos valores de referência para o uso da dinamometria. GED Gastroenterol Endosc Dig 1998;17:203-6.

6. Bland JN, Altman DG. Comparing methods of measurement: why plooting difference against standard method is misleading. Lancet 1995;346:1085-7.

7. Brown RS, Kumar KS, Russo MW, Kinkhabwala M, Rudow DL, Harren P, Lobritto S, Emond JC. Model of end-stage liver disease and Child-Turcotte-Pugh score as predictors of pretransplantation disease severity, posttransplantation outcome, and resource utilization in United Network for Organ Sharing status 2A patients. Liver Transpl 2002;8:278-84.

8. Correa MITD. Avaliação nutricional subjetiva. Rev Bras Nutr Clín 1998;13:68-73.

9. Davidson HIM, Richardson R, Sutherland D, Garden OJ. Macronutrient preference, dietary intake, and substrate oxidation among stable cirrhotic patients. Hepatology 1999;29:1380-6.

10. Detsky AS, Baker JP, Mendelson RA, Wolman SL, Wesson DE, Jeejeebhoy KN. Evaluating the accuracy of nutritional assessment techniques applied to hospitalized patients: methodology and comparison. JPEN J Parenter Enteral Nutr 1984;8:153-9.

11. Figueiredo FA, Dickson ER, Pasha TM, Kasparova P, Therneau T, Malinchoc M, DiCecco S, Francisco-Ziller N, Charlton M. Impact of nutritional status on outcome after liver transplantation. Transplantation 2000;70:1347-52.

12. Harris JA, Benedict FG. A biometric study of basal metabolism in man. Boston: Carnegie Institute of Washington; 1919. 266p.

13. Heyward VH, Sstolarczyk IM. Avaliação da composição corporal. São Paulo: Manole; 2000.

14. Hunt D, Rowlands BJ, Johnston D. Handgrip strength - a simple prognostic indicator in surgical patients. JPEN J Parenter Enteral Nutr 1985;9:701-4.

15. Jeejeebhoy KM, Hill GL, Owen OD. Nutritional and metabolism in patient care. Philadelphia: W.B. Saunders/Harcourt Brace Jovanovich; 1988. p.739-51.

16. Jeejeebhoy KM, Detsky AS, Baker JP. Assessment of nutritional status. JPEN J Parenter Enteral Nutr 1990;14(suppl):193s-6s.

17. Kondrup J, Muller MJ. Energy and protein requirements of patients with chronic liver disease. J Hepatol 1997;27:239-47.

18. Lowell JA. Nutritional assessment and therapy in patients requiring liver transplantation. Liver Transpl Surg 1996;2(5 suppl 1):79-88.

19. Madden AM, Morgan MY. Resting energy expenditure shoud be measured in patients with cirrosis, not predict. Hepatology 1999;30:655-64.

20. Mahan KL, Escott-Stump S. Krause Alimentos, nutrição e dietoterapia. $10^{a}$ edição. São Paulo: Roca; 2002.

21. McCullough AJ, Bugianesi E. Protein-calorie malnutrition and the etiology of cirrhosis. Am J Gastroenterol 1997;92:734.

22. McCullough AJ. Malnutrition and liver disease. Liver Transpl 2000;6(4 Suppl 1): S85-96.

23. McCullough AJ. Nutritional state and abnormal metabolism of nutrients. The American Association for the study of the Liver Diseases. Postgraduate course: newer aspects on alcohol, nutrition and hepatic encephalopathy, 1992; 204-44.
24. Mendenhall CL, Anderson S, Weesner RE, Goldberg SJ, Crolic KA. Protein-calorie malnutrition associated with alcoholic hepatitis. Veterans Administration Cooperative Study Group on Alcoholic Hepatitis. Am J Med 1984;76:211-22.

25. Merli M, Riggio O, Dally L. Does malnutrition affect survival in cirrhosis? PINC (Policentrica Italiana Nutrizione Cirrosi). Hepatology 1996;23:1041-6.

26. Miwa Y, Shiraki M, Kato M, Tajika M, Mohri H, Murakami N, Kato T, Ohnishi H, Morioku T, Muto Y, Moriwaki H. Improvement of fuel metabolism by nocturnal energy supplementation in patients with liver cirrhosis. Hepatol Res 2000;18:184-9.

27. Morgan MY. Nutrition and the liver. In: Millward-Sadler GH, Wright R, Arthur MJP editors. Wright's liver and biliary disease. 3rd edition. London: WB Saunders; 1992 p.107-73.

28. Muller MJ, Boker KHW, Selberg O. Are patients with liver cirrhosis hypermetabolic? Clin Nutr 1999;13:131.

29. National Research Council (U.S.). Subcommittee on Tenth Edition of the RDAs Recommended dietary allowances. 10th edition. Washington, DC: National Academy Press; 1989.

30. Parolin MB, Zaina FE, Lopes RW. Terapia nutricional no transplante hepático. Arq Gastroenterol 2002;39:114-22.

31. Pikul J, Sharpe M, Lowndes R, Ghent C. Degree of preoperative malnutrition is predictive of postoperative morbidity and mortality in liver transplantation recipients. Transplantation 1994;57:469-72.

32. Pugh R, Murray-Lyon I, Dawson J, Pietroni M, Williams R. Transection of the oesophagus for bleeding oesophagus varices. Br J Surg 1973;60:646-9.

33. Roongpisuthipong C, Sobhonslidsuk A, Nantiruj K, Songchitsomboom S. Nutritional assessment in various stages of liver cirrhosis. Nutrition 2001;17:761-5.

34. Schröder H, Covas MI, Marrugat J, Vila J, Pena A, Alcántara M, Masiá R. Use of a three-day estimated food record, a 72-hour recall and a food-frequency questionnaire for dietary assessment in Mediterranean Spanish population. Clin Nutr 2001;20:429-37.

35. Selberg O, Bottcher J, Tusch G, Pichlmayr R, Henkel E, Muller MJ. Identification of high-and low-risk patients before liver transplantation: a prospective cohort study of nutritional and metabolic parameters in 150 patients. Hepatology 1997;25:652-7.

36. Sherlock S, Dooley J. Diseases of the liver and biliary systems. 11th edition. Malden, MA: Blackwell Science; 2002

37. Shneeweiss B, Pammer J, Ratheiser K. Energy metabolism in acute hepatic failure Gastroenterology 1993;105:1515-21.

38. Shubhada NA, Flood K, Paranjothis S. Washington manual de terapêutica clínica 30a edição. Rio de Janeiro: Guanabara-Koogan; 2002.

39. Strauss E, Massuda HK. Avaliação nutricional em cirróticos. Arq Gastroenterol 1986;23:211-6

40. Tajika M, Kato M, Mohri H, Miwa Y, Kato T, Ohnishi H, Moriwaki H. Prognostic value of energy metabolism in patients with viral liver cirrhosis. Nutrition 2002;18:229-34.

41. Wahrlich V, Anjos LA. Aspectos históricos e metodológicos da medição e estimativa da taxa metabólica basal: uma revisão de literatura. Cad Saúde Pública 2001:17:801-17.

42. Waitzberg DL. Nutrição oral, enteral e parenteral na prática clínica. $3^{\mathrm{a}}$ edição São Paulo: Atheneu, 2000

43. Weir JB. New methods for calculating metabolic rate with special reference to protein metabolism. J Physiol 1949;109:1-9.

44. Zaina FE, Parolin MB, Lopes RW, Coelho JCU. Prevalência de desnutrição em candidatos a transplante hepático [resumo]. JBT J Bras Transpl 2003;6(1):50(A).

45. Zaina FE, Ribas MD. Comparação do estado nutricional nos três momentos do transplante hepático [resumo]. JBT J Bras Transpl 2003;6(1):50(A).

46. Zaina FE, Tiemann LF, Mello ACM, Lopes RW. Influência da ingestão dietética sobre o estado nutricional de adultos internados em um centro transplantador [resumo]. JBT J Bras Transpl 2003;6(1):50(A). 